DOI: $10.15593 / 2224-9982 / 2015.43 .06$

УДК 539.3

\author{
Н.М. Кочнева, А.В. Гуляев \\ АО «Научно-исследовательский институт полимерных материалов», \\ Пермь, Россия \\ ПРИМЕНЕНИЕ ФЕНОМЕНОЛОГИЧЕСКОЙ \\ НЕЛИНЕЙНОЙ МОДЕЛИ ДЛЯ ОПИСАНИЯ \\ ОСОБЕННОСТЕЙ МЕХАНИЧЕСКОГО ПОВЕДЕНИЯ \\ ДИСПЕРСНО-ВЫСОКОНАПОЛНЕННЫХ ПОЛИМЕРОВ
}

\begin{abstract}
Рассматривается нелинейная феноменологическая модель термовязкоупругого поведения дисперсно-наполненных полимерных материалов с массовой долей наполнения твердыми частицами более $87 \%$. Для учета структурных изменений полимера в процессе нагружения, обусловленных отслоениями матрицы от частиц наполнителя, в модель введен структурный параметр, определяемый эволюционным уравнением. Для идентификации параметров модели проведены большие экспериментальные исследования высоконаполненного полимера с массовой долей связующего 10,5 \%, наиболее часто используемого в конструкциях, в широком диапазоне температур $\left(-70 \ldots 120^{\circ} \mathrm{C}\right)$ и относительных скоростей деформирования $\left(10^{-5} \ldots 10^{0} \mathrm{c}^{-1}\right)$, которые достигаются в изделиях в процессе эксплуатации. Также получены экспериментальные зависимости величины объемных изменений, характеризующих изменение структуры материала в процессе его деформирования при температурах $\pm 50,20{ }^{\circ} \mathrm{C}$ и скоростях деформирования $10^{-4}, 10^{-3}, 10^{-2} \mathrm{c}^{-1}$. Получена зависимость коэффициента Пуассона от величины объемных изменений и уровня деформаций. На основании экспериментальных данных определены все константы нелинейной вязкоупругой модели. С использованием метода конечных элементов в рамках малых упругих деформаций рассчитано напряженно-деформированное состояние прочноскрепленных с корпусом изделий при действии температурной нагрузки. Показано, что с использованием нелинейной модели значения расчетных деформаций на свободной поверхности рассмотренных изделий возросли на 15-18\%, что существенно при назначении требований к механическим характеристикам полимера.

Ключевые слова: дисперсно-наполненные композиты, структурные повреждения, напряжение, деформация, объемные изменения.
\end{abstract}

\author{
N.M. Kochneva, A.V. Gulyaev
}

JSC "Research Institute of Polymeric Materials", Perm, Russian Federation

\title{
APPLICATION OF PHENOMENOLOGICAL NON-LINEAR MODEL FOR DESCRIPTION OF MECHANICAL BEHAVIOR PECULIARITIES OF DISPERSED HIGHLY-FILLED POLYMERS
}

The paper deals with the non-linear phenomenological model of thermo-viscoelastic behavior of dispersed-filled polymeric materials with solid loading mass fraction of more than $87 \%$. To take into account the polymer structural changes during the loading resulting from the peeling of the matrix from the filler particles, a structural parameter, determined by the evolution equation, was introduced to the 
model. For the identification of the model parameters the experimental studies on highly-filled polymer were conducted with the binder mass fraction of $10,5 \%$, more often used in constructions in the wide temperature range (minus $70-120^{\circ} \mathrm{C}$ ) and relative strain rates $\left(10^{-5}-10^{\circ} \mathrm{c}^{-1}\right)$, that are achieved in the products during their operation. Additionally the experimental dependences of the volumetric changes quantity, characterizing the material structure change during its straining at the temperatures $\pm 50,20^{\circ}$ $\mathrm{C}$ and straining rate $10^{-4}, 10^{-3}, 10^{-2} \mathrm{c}^{-1}$ were obtained. The dependence of Poisson ratio on the quantity of volumetric changes and the deformation level was obtained. On the basis of experimental results all constants of the non-linear viscoelastic model were determined. With the finite element method in the frames of small elastic strains a stressed-strained state of case-bonded products under thermal loading was calculated. It was shown that with the non-linear model the values of the calculated strain on the free surface of the products studied were increased by $15-18 \%$, that is significantly important while setting the polymer mechanical characteristic requirements. changes.

Keywords: dispersed highly-filled composites, structural damages, stress, strain, volumetric

В настоящее время широкое распространение в ракетной технике получили высоконаполненные полимерные материалы с дисперсным характером наполнения. Массовая доля наполнителя в таких материалах достигает $90 \%$, в результате чего линейное вязкоупругое поведение материала ограничивается деформированным состоянием (ДС) до $10 \%$. Современные же конструкции из дисперсно-наполненных полимерных материалов (ДПМ), как правило, эксплуатируются в широком температурном диапазоне $\pm 50{ }^{\circ} \mathrm{C}$. При этом максимальный уровень деформированного состояния ДПМ, используемого в этих конструкциях, достигает $20 \%$. Таким образом, эксплуатация полимерного материала в изделиях происходит в нелинейной области деформирования. Эффективный метод решения (метод аппроксимаций) задач нелинейной теории термовязкоупругости предложен в работе [1], но там еще не рассматривалось влияние структурных изменений на напряженнодеформированное состояние материала.

Существует большое количество как структурных, так и феноменологических моделей, позволяющих учесть взаимодействие твердых частиц наполнителя с матрицей при деформировании. Наиболее полно результаты исследования этого взаимодействия представлены в работах [2-4]. Следует отметить также работы [5, 6], где для описания механического поведения наполненных полимерных материалов применена эндохронная нелинейная теория термовязкоупругости. На рис. 1 показаны теоретические зависимости, полученные с использованием уравнений линейной вязкоупругости, и экспериментальные зависимости напряжений от деформаций в случае одноосного растяжения лабораторного образца-лопаточки при относительной скорости деформирования $\sim 10^{-3} \mathrm{c}^{-1}$ и разных температурах. 


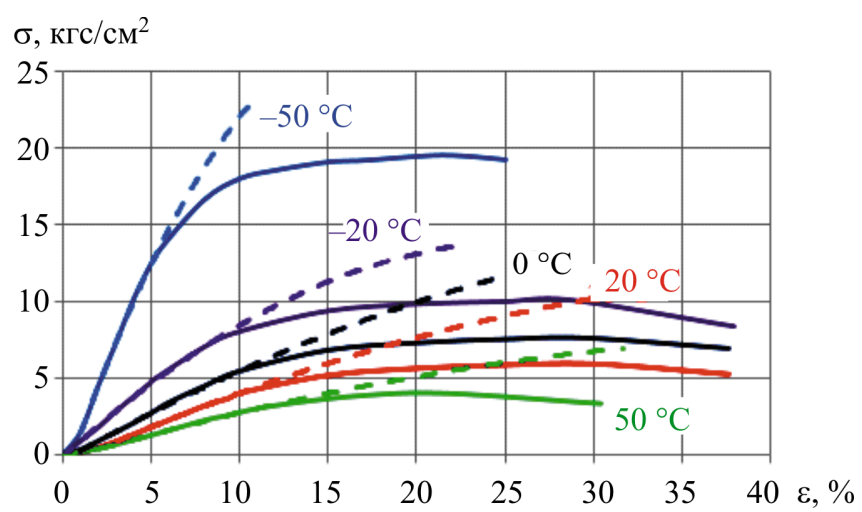

Рис. 1. Теоретические (пунктирные линии) и экспериментальные (сплошные линии) диаграммы растяжения при относительной скорости деформирования $\sim 10^{-3} \mathrm{c}^{-1}$ образца-лопаточки

Некоторые результаты уже были изложены в работах [7-9]. Ниже приводится дальнейшее развитие модели.

Для описания термовязкоупругого поведения наполненных полимерных материалов на этапе активного нагружения при малых деформациях за основу взята модель $[10,11]$

$$
\hat{\sigma}(\mathrm{t})=M(Z) \cdot 2 \mu[\dot{\varepsilon}, T(t)]\left\{\left(\varepsilon_{i j}-\frac{\theta}{3} \delta_{i j}\right)\right\}+\sigma \delta_{i j},
$$

где $M(Z)$ - функция структурного параметра $Z ; \mu[\dot{\varepsilon}, T(\mathrm{t})]$ - модуль сдвига, зависящий от температурно-временных условий нагружения; $\theta$ - объемные изменения материала без температурной составляющей; $\delta_{\mathrm{ij}}-$ символ Кронекера; $\sigma$ - среднее напряжение, определяемое выражением

$$
\sigma=B \cdot\left[\theta-F\left(\sigma, Z, I_{\gamma}\right)\right]
$$

где $B$ - модуль объемного сжатия; $F\left(\sigma, Z, I_{\gamma}\right)$ - нелинейная функция, учитывающая объемные изменения в зависимости от меры сдвиговых деформаций $I_{\gamma}$, структурных изменений и среднего напряжения.

Согласно работе [1] функция $F\left(\sigma, Z, I_{\gamma}\right)$ зависит от меры сдвиговых деформаций и функции среднего напряжения:

$$
F\left(\sigma, Z, I_{\gamma}\right)=B_{1} \cdot I_{\gamma}^{n} \cdot Z \cdot \exp \left[B_{2} \cdot\left(\sigma-\mu[T, t] \cdot I_{\gamma} / \sqrt{3}\right],\right.
$$


где $B_{1}, n, B_{2}$ - константы материала, определяемые из эксперимента с регистрацией объемных изменений лабораторного образца при нагружении.

Уравнение для функции $M(Z)$ принималось в виде

$$
M(Z)=\left(1-k_{z} Z-k_{z} Z^{2}\right)
$$

где $k_{z}$ - параметр модели, определяющий ее нелинейное поведение. Для структурного параметра $Z$ принято уравнение

$$
Z=\max \left\{[\chi(T)] I_{\gamma}\right\},
$$

где $\chi(T)$ - безразмерный параметр, определяемый как отношение предельной деформации при приведенной температуре к предельной деформации при текущей температуре.

В качестве меры интенсивности сдвиговых деформаций взято выражение

$$
I_{\gamma}=\frac{\sqrt{2}}{2(1+v(\theta, \varepsilon))} \sqrt{\left(\varepsilon_{1}-\varepsilon_{2}\right)^{2}+\left(\varepsilon_{2}-\varepsilon_{3}\right)^{2}+\left(\varepsilon_{3}-\varepsilon_{1}\right)^{2}},
$$

где $v$ - коэффициент Пуассона, зависящий от величины объемных изменений; $\varepsilon_{1}, \varepsilon_{2}, \varepsilon_{3}$ - главные напряжения тензора деформации.

Для оператора модуля сдвига $\mu(T, t)$ принят интеграл из классической теории вязкоупругости [12]:

$$
\mu(T, t) \cdot \varphi(t)=\int_{0}^{t} K\left(t^{\prime}-\tau^{\prime}\right) \mathrm{d} \varphi\left(\tau^{\prime}\right)
$$

где $K$ - функция ползучести; $t^{\prime}, \tau^{\prime}-$ приведенные значения времени, зависящие от функции температурно-временного смещения $a_{T}[T]$; $\varphi(t)$ - произвольная функция, зависящая от времени.

В качестве функции для коэффициента температурно-временного смещения $a_{T}[T]$ использовалась функция

$$
a_{T}[T]=10^{A\left[\frac{1}{T(t)}-\frac{1}{293}\right]},
$$

где $A$ - константа материала, определяемая из опытов на ползучесть или релаксацию. 
Функция ползучести $K\left(t^{\prime}\right)$ принималась в виде, аналогичном [13]

$$
K\left(t^{\prime}\right)=E_{\infty}+\left(10^{5}-E_{\infty}\right) \cdot \exp \left[-\left(\frac{\mathrm{t}^{\prime}}{\vartheta}\right)^{\alpha}\right]
$$

где $E_{\infty}, E_{0}, \vartheta, \alpha$ - константы материала, определяемые из опыта на ползучесть.

В данной работе для полного определения материальных констант модели и механических характеристик были проведены и использованы следующие лабораторные эксперименты на растяжение и сжатие:

- испытания на ползучесть в широком температурном диапазоне $-70 . .120^{\circ} \mathrm{C}$;

- испытания на одноосное растяжение образца-лопаточки в широком диапазоне относительных скоростей деформирования $10^{-5}-10^{0} \mathrm{c}^{-1}$ и широком температурном диапазоне; столбиков;

- испытания на одноосное и всестороннее сжатие образцов-

- дилатометрические исследования образца-лопаточки при одноосном растяжении в широком диапазоне температур и относительных скоростей деформирования;

- испытания образца лопаточки на одноосное растяжение при избыточном давлении до 10 МПа.

Результаты лабораторных исследований образцов-лопаточек из материала с массовой долей наполнения твердыми частицами 89\% представлены на рис. 2-4.

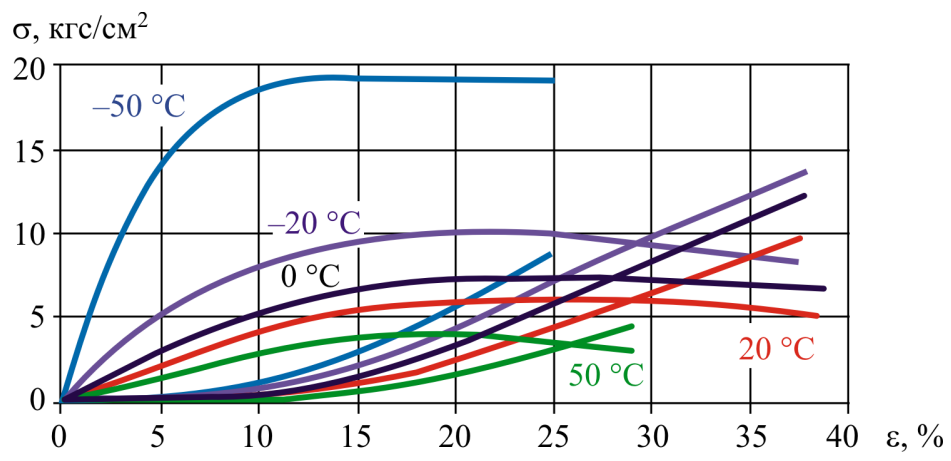

Рис. 2. Диаграммы растяжения и дилатансии при относительной скорости деформирования образца-лопаточки $\sim 10^{-3} \mathrm{c}^{-1}$ 


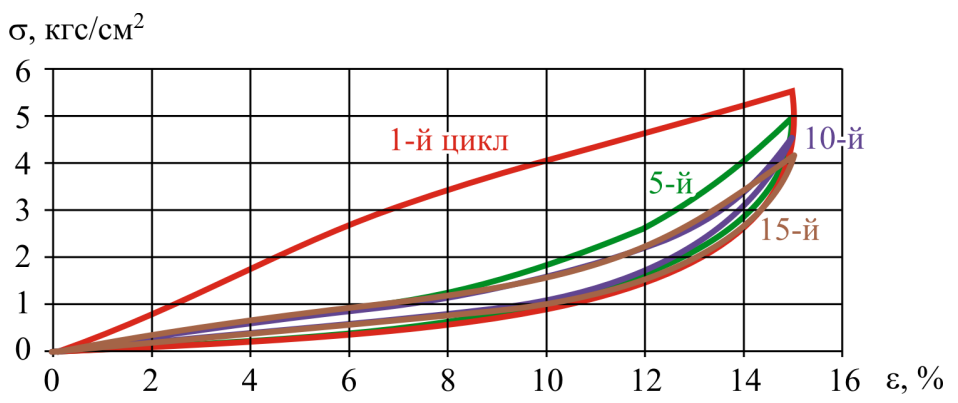

Рис. 3. Циклическое нагружение образца-лопаточки до деформации $15 \%$ при относительной скорости деформирования $\sim 10^{-3} \mathrm{c}^{-1}$ и температуре $20^{\circ} \mathrm{C}$

$\sigma, \kappa \Gamma \mathrm{c} / \mathrm{cm}^{2}$

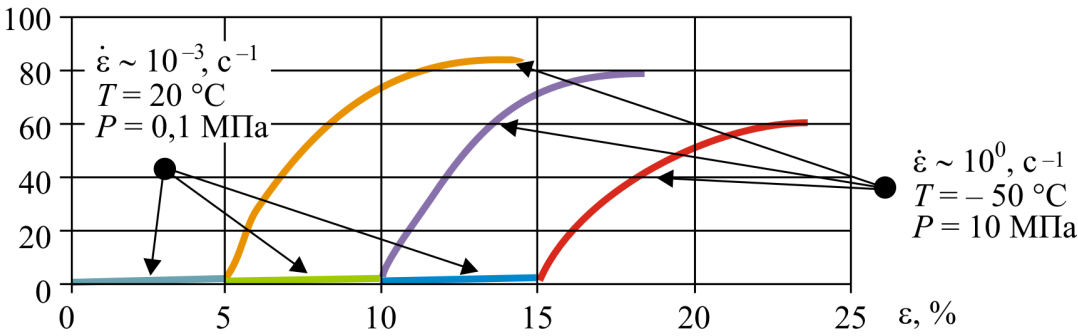

Рис. 4. Испытания образцов-лопаточек при ступенчатом трехосном режиме нагружения

В результате испытаний на ползучесть в линейной области деформирования получены следующие значения констант функций $K\left(t^{\prime}\right)$ и $a_{T}[T]: E_{\infty}=0,7$ МПа, $\vartheta=10^{-47,44} \mathrm{c}, \alpha=0,019, A=6210 \mathrm{~K}$. Из испытаний на одноосное растяжение получены значения констант нелинейной модели, приведенные ниже.

Значения констант нелинейной модели

\begin{tabular}{|c|c|c|c|c|c|c|}
\hline $\begin{array}{l}\text { Коэффициент } \\
\text { Пуассона, v }\end{array}$ & 0,469 & 0,437 & 0,405 & 0,378 & 0,360 & 0,346 \\
\hline$\varepsilon, \%$ & 10 & 15 & 20 & 25 & 30 & 35 \\
\hline $\begin{array}{c}\text { Объемные изменения } \\
\theta, \%\end{array}$ & 0,62 & 1,90 & 3,80 & 6,10 & 8,60 & 10,80 \\
\hline$\sigma, \kappa г \mathrm{c} / \mathrm{cm}^{2}$ & 1,60 & 1,8 & 1,87 & 1,90 & 1,97 & 2,03 \\
\hline$B_{1}$ & \multicolumn{6}{|c|}{0,767} \\
\hline$\Pi$ & \multicolumn{6}{|c|}{0,867} \\
\hline$B_{2}$ & \multicolumn{6}{|c|}{1,352} \\
\hline$k_{z}$ & \multicolumn{6}{|c|}{0,4} \\
\hline
\end{tabular}


Зависимость функции $\chi(T)$ от температуры

\begin{tabular}{|c|c|c|c|c|c|c|c|c|}
\hline$T,{ }^{\circ} \mathrm{C}$ & -70 & -50 & -40 & -20 & 0 & 20 & 50 & 70 \\
\hline$\chi(T)$ & 1,33 & 1,10 & 0,98 & 0,93 & 0,93 & 1,00 & 1,11 & 1,20 \\
\hline
\end{tabular}

Вариационное уравнение для решения задачи имеет вид

$$
\Phi(\{\varepsilon\})=\frac{1}{2} \iiint_{\mathrm{V}}\left(\hat{\sigma} \cdot \delta \hat{\varepsilon}+\left[\theta-\alpha_{T} \Delta T-\frac{\sigma}{B}\right] \delta \sigma\right) \mathrm{d} V-\iint_{\mathrm{S}_{\sigma}} \vec{P} \cdot \delta u \cdot \mathrm{d} S .
$$

В связи со сложной геометрией рассматриваемых конструкций решение краевой задачи термосилового нагружения осуществлялось приближенными методами инженерного исследования, поэтому для определения неизвестного распределения температуры и соответствующего напряженно-деформированного состояния использовались вариационные уравнения, решаемые с помощью метода конечных элементов [14].

Вышеприведенная математическая модель была использована для определения напряженно-деформированного состояния двух прочно скрепленных со стальным корпусом изделий (рис. 5) из наполненного полимера при их термостатировании при температуре от +50 до $-50{ }^{\circ} \mathrm{C}$. При этом предполагалось, что равновесная температура изделий составляет $50^{\circ} \mathrm{C}$.

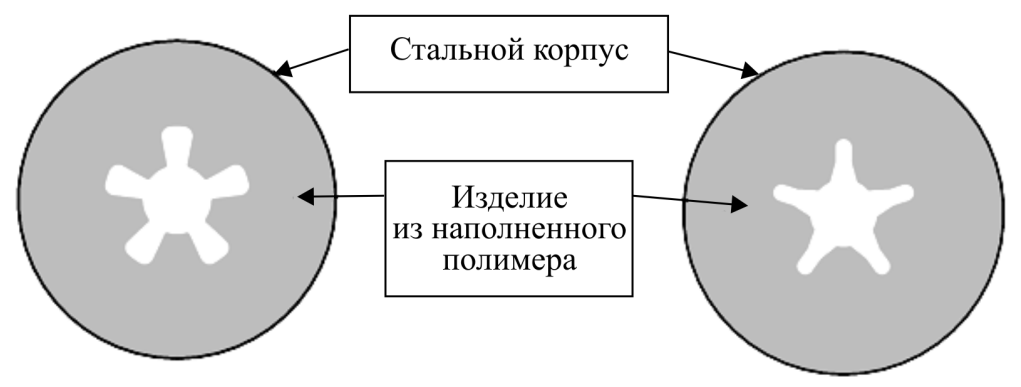

Рис. 5. Поперечные сечения прочно скрепленных с корпусом изделий из дисперсно-высоконаполненного полимера

В результате решения задачи термовязкоупругости получены значения максимальных деформаций в изделии № 1 (рис. 6, a): в рамках линейной теории вязкоупругости - 23,3\%, в рамках нелинейной модели $-27,5 \%$. Для изделия № 2 (рис. $6, \sigma)$ в рамках линейной теории получено 24,9\%, в рамках нелинейной $-28,7 \%$. 

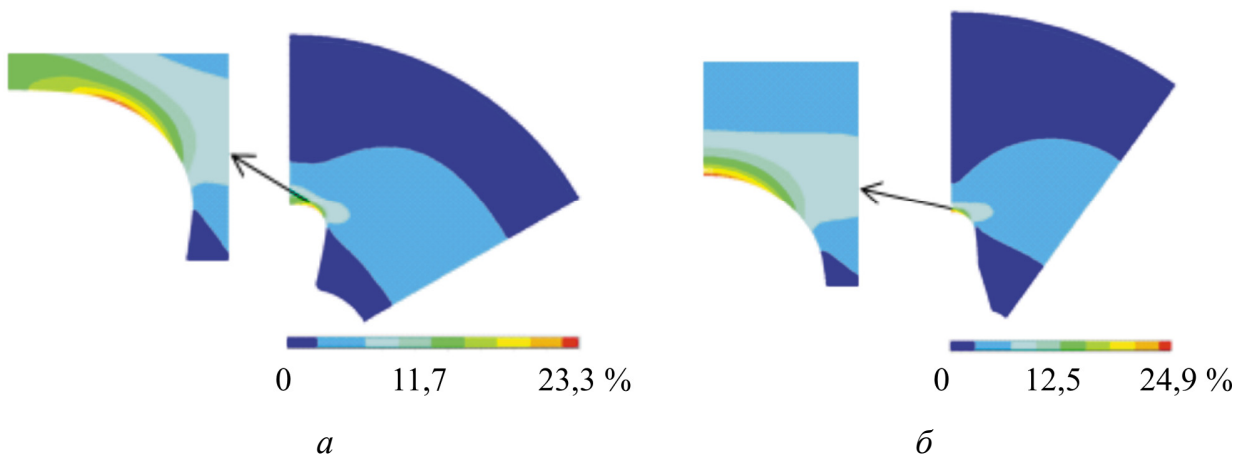

Рис. 6. Распределение максимальных деформаций в изделиях при охлаждении от +50 до $-50{ }^{\circ} \mathrm{C}$

Более высокие значения деформаций, полученных из нелинейной модели, приводят к необходимости ее использования при оценке прочности разрабатываемых конструкций.

\section{Библиографический список}

1. Ильюшин А.А., Победря Б.Е. Основы математической теории термовязкоупругости. - М.: Наука, 1970. - 280 с.

2. Структурные механизмы формирования механических свойств зернистых полимерных композитов / В.В. Мошев, А.Л. Свистков, О.К. Гаришин [и др.]. - Екатеринбург: Изд-во УрО РАН, 1997. - 508 с.

3. Farris R.J. The influence of vacuole formation on the response and failure of filled elastomers // J. Trans. Soc. Rheol. - 1968. - Vol. 12, № 2. P. 315-334.

4. Naviere R. An extension of the time-temperature superposition principle to non-linear viscoelastic solids // Int. J. Solids and Structures. 2006. - № 43. - P. 5295-5306.

5. Апетьян В.Э., Быков Д.Л. Анализ немонотонной зависимости напряжений от деформаций в вязкоупругих средах // Механика твердого тела. - 2004. - № 4. - С. 106-115.

6. Быков Д.Л., Коновалов Д.Н. Использование структурных составляющих удельной работы внутренних сил для оценки прочности вязкоупругих конструкций в зонах локальной концентрации напряжений // Механика твердого тела. - 2007. - № 6. - С. 84-93.

7. Кочнева Н.М., Гуляев А.В. Применение нелинейной модели вязкоупругого поведения при расчете деформированного состояния 
конструкций // Tp. XXXIII Bсерос. конф. по проблемам науки и технологий. - 2013. - Т. 2. - С. 51-53.

8. Методика расчета и назначение режимов термостатирования изделий, эксплуатирующихся в широком температурном диапазоне, для подтверждения длительных сроков их хранения / Н.М. Кочнева, А.В. Гуляев, Н.Е. Пшеницына, С.В. Ямалитдинова // Материалы XXXIV Bcepoc. конф., посвященной 90-летию со дня рождения акад. В.П. Макеева. - М.: Изд-во РАН, 2014. - Т. 2. - С. 155-160.

9. Кочнева Н.М., Гуляев А.В. Применение феноменологической, нелинейной модели для описания особенностей механического поведения дисперсно-высоконаполненных полимеров // Сб. тр. ХІ Всерос. съезда по фундаментальным проблемам теоретической и прикладной механики. - Казань: Изд-во Казан. федер. ун-та, 2015. - С. 2040-2042.

10. Адамов А.А. Неизотермическое деформирование элементов конструкции из нелинейного дисперсно-наполненного эластомера // Механика композиционных материалов и конструкций. - 1999. - Т. 5, № 2. - С. 101-107.

11. Адамов А.А. К построению модели вязкоупругого поведения наполненных эластомеров с учетом структурных изменений // Исследование по механике материалов и конструкций. - Свердловск: Изд-во УрО АН СССР, 1988. - С. 4-6.

12. Москвитин В.В. Сопротивление вязкоупругих материалов (применительно к зарядам ракетных двигателей на твердом топливе). М.: Наука, 1972. - 328 с.

13. Слонимский Г.Л., Роговина Л.З. Определение механических характеристик полимерного материала по релаксации напряжения при постоянной деформации // Высокомолекулярные соединения. - 1964. Т. 6, № 4. - С. 620-623.

14. Зенкевич О. Метод конечных элементов в технике. - М.: Мир, 1975. $-541 \mathrm{c}$.

\section{References}

1. Ilyushin A.A., Pobedrya B.E. Osnovy matematicheskoy teorii termovyazko-uprugosti [The foundations of mathematical theory of thermoviscoelastisity]. Moscow: Nauka, 1970. 280 p.

2. Moshev V.V., Svistkov A.L., Garishin O.K. [et al.]. Strukturnye mekhanizmy formirovaniya mekhanicheskikh svoystv zernistykh polimer- 
nykh kompozitov [Structural mechanisms of forming mechanical properties of particulate polymeric composites]. Ekaterinburg: Uralskoe otdelenie Rossiyskoy akademii nauk, 1997. 508 p.

3. Farris R.J. The influence of vacuole formation on the response and failure of filled elastomers. J. Trans. Soc. Rheol., 1968, vol. 12, no. 2, pp. 315-334.

4. Naviere R. An extension of the time-temperature superposition principle to non-linear viscoelastic solids. Int. J. Solids and Structures, 2006, no. 43, pp. 5295-5306.

5. Apetyan V.E., Bykov D.L. Analiz nemonotonnoy zavisimosti napryazheniy ot deformatsiy $\mathrm{v}$ vyazkouprugikh sredakh [Analysis of nonmonotonous dependence of stresses on strains in viscoelastic medium]. Mekhanika tverdogo tela, 2004, no. 4, pp. 106-115.

6. Bykov D.L., Konovalov D.N. Ispolzovanie strukturnykh sostavlyayushchikh udelnoy raboty vnutrennikh sil dlya otsenki prochnosti vyazkouprugikh konstruktsiy $\mathrm{v}$ zonakh lokalnoy kontsentratsii napryazheniy [The application of structural constituents of the specific work for evaluation of viscoelastic strength in zones of local stress concentration]. Mekhanika tverdogo tela, 2007, no. 6, pp. 84-93.

7. Kochneva N.M., Gulyaev A.V. Primenenie nelineynoy modeli vyazkouprugogo povedeniya pri raschete deformirovannogo sostoyaniya konstruktsiy [Application of non-linear model of viscoelastic behavior while calculating the construction strained state]. Trudy XXXIII Vserossiyskoy konferentsii po problemam nauki $i$ tekhnologiy, 2013, vol. 2, pp. 51-53.

8. Kochneva N.M., Gulyaev A.V., Pshenitsyna N.E., Yamalitdinova S.V. Metodika rascheta i naznachenie rezhimov termostatirovaniya izdeliy, ekspluatiruyushchikhsya $\mathrm{v}$ shirokom temperaturnom diapazone dlya podtverzhdeniya dlitelnykh srokov ikh khraneniya [Calculation methods and setting the product termostating mode, operating in a wide temperature range for the verification of their long-term storage life]. Materialy XXXIV Vserossiyskoy konferentsii, posvyashchennoy 90-letiyu so dnya rozhdeniya akademika V.P. Makeeva, 2014, vol. 2, pp. 155-160.

9. Kochneva N.M., Gulyaev A.V. Primenenie fenomenologicheskoy, nelineynoy modeli dlya opisaniya osobennostey mekhanicheskogo povedeniya dispersno vysokonapolnennykh polimerov [Application of phe- 
nomenological non-linear model for description of peculiarities of dispersed-filled polymers mechanical behavior]. Sbornik trudov XI Vserossiyskogo sezda po fundamentalnym problemam teoreticheskoy $i$ prikladnoy mekhaniki, 2015, pp. 2040-2042.

10. Adamov A.A. Neizotermicheskoe deformirovanie elementov konstruktsii iz nelineynogo dispersno-napolnennogo elastomera [Non-isotermal deformation of construction elements from non-linear dispersed filled elastomer]. Mekhanika kompozitsionnykh materialov i konstruktsiy, 1999, vol. 5, no. 2, pp. 101-107.

11. Adamov A.A. K postroeniyu modeli vyazkouprugogo povedeniya napolnennykh elastomerov $\mathrm{s}$ uchetom strukturnykh izmeneniy [To the construction of model of filled elastomers taking into account the structural changes]. Issledovyanie po mekhanike materialov $i$ konstruktsiy. Sverdlovsk: Uralskoe otdelenie Akademii nauk SSSR, 1988, pp. 4-6.

12. Moskvitin V.V. Soprotivlenie vyazkouprugikh materialov (primenitelno $\mathrm{k}$ zaryadam raketnykh dvigateley na tverdom toplive) [Resistance of viscoelastic materials (relative to SRM chargers)]. Moscow: Nauka, 1972. 328 p.

13. Slonimskiy G.L., Rogovina L.Z. Opredelenie mekhanicheskikh kharakteristik polimernogo materiala po relaksatsii napryazheniya pri postoyannoy deformatsii [Determination of polymeric material mechanical properties according to stress relaxation at constant strain]. Vysokomolekulyarnye soedineniya, 1964 , vol. 6 , no. 4, pp. 620-623.

14. Zenkevich O. Metod konechnykh elementov $\mathrm{v}$ tekhnike [Finite element method in engineering]. Moscow: Mir, 1975. 541 p.

\section{Об авторах}

Кочнева Наталья Михайловна (Пермь, Россия) - кандидат технических наук, начальник отдела прочности АО «Научно-исследовательский институт полимерных материалов» $(614113$, г. Пермь, ул. Чистопольская, д. 16, e-mail: niipm@pi.ccl.ru).

Гуляев Алексей Владимирович (Пермь, Россия) - начальник лаборатории расчетных методов оценки прочности АО «Научно-исследовательский институт полимерных материалов» (614113, г. Пермь, ул. Чистопольская, д. 16, e-mail: GulyaevAV@inbox.ru). 


\section{About the authors}

Natalya M. Kochneva (Perm, Russian Federation) - Ph. D. in Technical Sciences, Head of the Strength Department, JSC "Research Institute of Polymeric Materials" (16, Chistopolskaya st., Perm, 614113, Russian Federation, niipm@pi.ccl.ru).

Aleksey V. Gulyaev (Perm, Russian Federation) - Head of the Strength Evaluation Calculation Methods Laboratory, JSC "Research Institute of Polymeric Materials" (16, Chistopolskaya st., Perm, 614113, Russian Federation, GulyaevAV@inbox.ru).

Получено 27.10.2015 